\title{
Right Ventricular Myocardial Ischemia with Arrhythmia in an Asphyxiated Newborn
}

\author{
Anne Lee Solevåg, MD, MSc, PhD ${ }^{1,2,3}$ Georg M. Schmölzer, MD, PhD ${ }^{1,2}$ \\ Po-Yin Cheung, MBBS, FRCP, PhD (Can\&Edin) ${ }^{1,2}$
}

${ }^{1}$ Centre for the Studies of Asphyxia and Resuscitation, Neonatal Intensive Care Unit, Royal Alexandra Hospital, Edmonton, Alberta, Canada

2 Department of Pediatrics, University of Alberta, Edmonton, Alberta, Canada

${ }^{3}$ Department of Pediatric and Adolescent Medicine, Akershus University Hospital, Lørenskog, Norway

Am J Perinatol Rep 2016;6:e203-e205.
Address for correspondence Po-Yin Cheung, MBBS, FRCP (Can\&Edin), $\mathrm{PhD}$, Centre for the Studies of Asphyxia and Resuscitation, Neonatal Research Unit, Royal Alexandra Hospital, 10240 Kingsway Avenue NW, Edmonton, AB T5H 3V9, Canada (e-mail: poyin@ualberta.ca).

\begin{abstract}
Keywords

- newborn

- asphyxia

- myocardial infarction

- arrhythmia

Background Infant and neonatal myocardial infarction (MI) has been described in association with congenital heart disease, coronary artery abnormalities, myocarditis, and tumors. $\mathrm{Ml}$ in the perinatal period in a structurally normal heart and with ventricular arrhythmia as a presenting feature has not been thoroughly described. Published case reports describe treatment methods extrapolated from adult MI. However, due to the rare occurrence, the most appropriate acute treatment for both $\mathrm{MI}$ and ventricular arrhythmia in newborn infants remains unknown.

Case A male term infant with perinatal asphyxia and need for extensive cardiopulmonary resuscitation at birth had ventricular tachyarrhythmia and ST-elevations on electrocardiogram. Four hours after birth, he died from cardiogenic failure. A thrombus at the right coronary artery was found on autopsy.

Conclusion $\mathrm{MI}$ in the perinatal period in a structurally normal heart is very rare and mortality is high. Although acute treatments extrapolated from adult MI has been described to result in favorable outcomes in newborn infants, guidelines are lacking on how to manage acute $\mathrm{MI}$ and associated ventricular arrhythmia.
\end{abstract}

Infant and neonatal myocardial infarction (MI) has been described in association with congenital heart disease, coronary artery abnormalities, myocarditis and tumors. MI in the perinatal period in a structurally normal heart and with ventricular arrhythmia as a presenting feature has not been thoroughly described, and the most appropriate acute treatment in newborn infants remains unknown.

\section{Case}

A male term infant was born via vaginal delivery to a 34-yearold primigravida after a pregnancy without identifiable risk factors. Cardiotocogram showed fetal tachycardia before de- livery and meconium stained amniotic fluid was present. The Apgar scores were 2-1-0 at 1, 5, and 10 minutes, respectively, and the umbilical cord $\mathrm{pH}$ 7.07. The newborn required extensive cardiopulmonary resuscitation (CPR) for $15 \mathrm{mi}-$ nutes at birth. After return of spontaneous circulation, a bigeminy cardiac rhythm was observed. The newborn remained hypotensive despite aggressive catecholamine treatment with epinephrine and dopamine at 1 and $10 \mu \mathrm{g} / \mathrm{kg} / \mathrm{min}$, respectively.

A three-lead electrocardiogram (ECG) showed arrhythmia interchanging among bigeminy, trigeminy, and ventricular arrhythmia with marked ST-elevations (-Fig. 1). The condition deteriorated with recurrent cardiac arrest and received

February 21, 2016 accepted after revision April 25, 2016
DOI http://dx.doi.org/

10.1055/s-0036-1584279. ISSN 2157-6998.
Copyright $\odot 2016$ by Thieme Medical Publishers, Inc., 333 Seventh Avenue, New York, NY 10001, USA. Tel: +1(212) 584-4662.
License terms

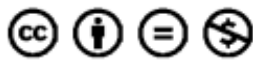




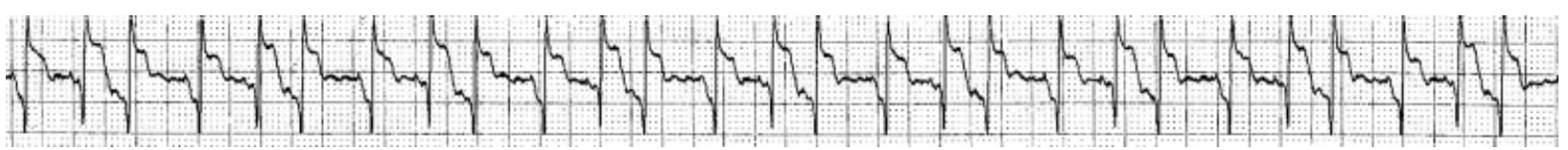

Fig. 1 A three-lead electrocardiogram showing marked ST-elevation as well as a striking trigeminy rhythm.

bradyarrhythmia and active treatment was withdrawn 4 hours after birth. The newborn died shortly after. Laboratory investigations at 2 hours of age showed troponin I $0.32 \mu \mathrm{g} / \mathrm{L}$ (ref. $<0.15 \mu \mathrm{g} / \mathrm{L}$ ), hemoglobin $112 \mathrm{~g} / \mathrm{L}$, and coagulopathy. At autopsy, a loose thrombus was found at the right coronary artery (RCA), with interstitial edema in the tissue of an otherwise structurally normal heart.

\section{Discussion}

In this highly unusual case, the finding of thrombosis at the RCA is interesting, as it might have caused myocardial ischemia or MI leading to arrhythmias and cardiogenic failure.

Similar to adults, MI in newborns is diagnosed with a combination of cardiac serum biomarkers, ECG, echocardiography, and cardiac angiography. ${ }^{1}$ In our patient, echocardiography could have been very useful, not only to rule out congenital heart disease but also to assess ventricular function and regional hypokinesis, and guide treatment. Also, the coronary artery occlusion could potentially have been diagnosed with Doppler echocardiography. We measured an elevated troponin I, but the very unstable condition of the infant precluded the acquisition of a 12-lead ECG, echocardiography, and angiography. Thus, further classification of the myocardial ischemia other than the presence of arrhythmia and ST-elevation was not possible.

We do not know for sure if fetal distress with meconium aspiration and hypoxia/asphyxia or the RCA thrombus was the primary pathology and lead to the initial need for CPR. The primary event could have been respiratory failure leading to hypoxia, which then might have precipitated the RCA thrombus formation and the arrhythmia. Complications of perinatal asphyxia include increased myocardial oxygen demand, anemia, and disseminated intravascular coagulopathy, and the association between perinatal asphyxia and myocardial ischemia has been described previously. ${ }^{2}$ However, in this case, the RCA thrombus could have been the primary event as well. We can only speculate on the origin of the RCA thrombus, but in the presence of open fetal vascular shunts, it could have originated from an embolus on the venous or arterial side of the circulation. It might also have originated in the RCA itself.

Considering the fact that the right ventricle is dominant immediately after birth, and that perinatal asphyxia is commonly associated with persistent pulmonary hypertension, occlusion of the RCA may explain the fulminant clinical course. The short clinical course with early death could explain the minimal histological changes of MI and the modest increase in the serum troponin I level.

Even though histological sign of myocardial ischemia is not an uncommon finding on autopsy of infants who died in the neonatal intensive care unit, ${ }^{3}$ clinical MI in the perinatal period is very rare. ${ }^{4}$ In our tertiary perinatal center, admitting approximately 1,500 infants to the neonatal intensive care unit annually, this is the first known case of MI in the perinatal period.

In addition to perinatal asphyxia, infant MI has been described in association with congenital heart disease, coronary artery abnormalities, myocarditis, tumors, ${ }^{3}$ and coagulation disorders. ${ }^{5}$ Histological findings range from inflammation to calcification and fibrosis. ${ }^{3}$ Acute treatments described include oxygen, angiotensin-converting enzyme inhibitor and aspirin, ${ }^{6}$ nitroglycerine, extracorporeal membrane oxygenation, ${ }^{4,7}$ and intravenous thrombolysis (tissue plasminogen activator). ${ }^{1}$ For the associated hypotension, that is, low cardiac output syndrome, inotropes, diuretics, and levosimendan, a calcium-sensitizing drug with vasodilating properties has been described to be effective. $^{2}$ However, these treatments are all essentially extrapolated from adult MI. Guidelines are lacking on how to manage acute neonatal MI and mortality remains high, ${ }^{8}$ as illustrated by this rare case history of death from cardiogenic failure the first hour of life. The associated ventricular arrhythmia in this infant is also an infrequently occurring symptom in the immediate postnatal period. Again, treatment options except for hemodynamic support may be scarce, although the use of lidocaine infusion ${ }^{9}$ and cardioversion $^{9,10}$ has been described.

Farooqi et $\mathrm{al}^{8}$ presented a case history and review of neonatal MI and concluded that due to the rare occurrence, it is unknown whether certain etiologies of MI are associated with particularly poor outcome, or if poor outcomes are mostly attributed to delays in identification and institution of treatment. In our patient, the fulminant course and early death precluded extensive diagnostic evaluations, but postmortem examinations suggested right ventricular myocardial ischemia due to a thrombus in the RCA.

\section{Conclusion}

$\mathrm{MI}$ in the perinatal period is rare but has been described with a wide array of potential causes and treatments. MI in a structurally normal heart is even more rare, and ventricular arrhythmia as the presenting feature has not been clearly described in the literature. Besides providing supportive treatment, clinicians may find themselves lacking guidelines for a targeted approach when faced with acute MI in newborn infants. The same applies to ventricular arrhythmia, which is also very rare in the perinatal period.

\section{Conflict of Interest}

The authors declare no conflict of interest. 


\section{Acknowledgments}

We would like to thank the parents of the infant described in this case report for providing their consent for the publication of the history. A.L.S. is supported by the Canadian Institutes of Health Research (operating grant MOP-CIA-299111 to PYC and travel award to A.L.S.) and the South-Eastern Norway Regional Health Authority. G.M.S. is supported by a Heart and Stroke Foundation/University of Alberta Professorship for Neonatal Resuscitation and by a Heart and Stroke Foundation of Canada Research Scholarship.

\section{References}

1 Haubner BJ, Schneider J, Schweigmann U, et al. Functional recovery of a human neonatal heart after severe myocardial infarction. Circ Res 2016;118(2):216-221

2 Caruso E, Di Pino A, Poli D, Manuri L, Guccione P. Erythrocytosis and severe asphyxia: two different causes of neonatal myocardial infarction. Cardiol Young 2014;24(1): 178-181
3 Bamber AR, Pryce J, Cook A, Ashworth M, Sebire NJ. Myocardial necrosis and infarction in newborns and infants. Forensic Sci Med Pathol 2013;9(4):521-527

4 Bulbul ZR, Rosenthal DN, Kleinman CS. Myocardial infarction in the perinatal period secondary to maternal cocaine abuse. A case report and literature review. Arch Pediatr Adolesc Med 1994; 148(10):1092-1096

5 Cesna S, Eicken A, Juenger H, Hess J. Successful treatment of a newborn with acute myocardial infarction on the first day of life. Pediatr Cardiol 2013;34(8):1868-1870

6 Abbal J, Paranon S, Brierre G, Dulac Y, Casper C, Acar P. Myocardial infarction in a newborn from a diabetic mother. Cardiol Young 2010;20(4):451-454

7 Ferns S, Khan M, Firmin R, Peek G, Bu'Lock F. Neonatal myocardial infarction and the role of extracorporeal membrane oxygenation. Arch Dis Child Fetal Neonatal Ed 2009;94(1):F54-F57

8 Farooqi KM, Sutton N, Weinstein S, Menegus M, Spindola-Franco $\mathrm{H}$, Pass RH. Neonatal myocardial infarction: case report and review of the literature. Congenit Heart Dis 2012;7(6):E97-E102

9 Nirupam N, Nangia S, Saili A. Ventricular tachycardia due to perinatal asphyxia. Indian Pediatr 2014;51(3):227-228

10 Heinonen KM. Rare procedures during delivery room resuscitation -cardioversion of ventricular tachycardia in an asphyctic neonate. Intensive Care Med 1992;18(8):491-492 